\title{
NEAR-INFRARED OBSERVATIONS OF A REDSHIFT 5.34 GALAXY: FURTHER EVIDENCE FOR DUST ABSORPTION IN THE EARLY UNIVERSE ${ }^{1}$
}

\author{
L. Armus,${ }^{2,3}$ K. Matthews, ${ }^{3}$ G. Neugebauer, ${ }^{3}$ and B. T. Solfer ${ }^{3}$ \\ Received 1998 April 7; accepted 1998 June 17; published 1998 September 9
}

\begin{abstract}
Imaging at 1.25 and $2.20 \mu \mathrm{m}$ has been obtained of the field containing the galaxy (RD1) found at redshift $z=5.34$ by Dey et al. This galaxy has been detected at $1.25 \mu \mathrm{m}$, while the lower redshift $(z=4.02)$ galaxy also found in the same field by Dey et al. was detected at both 1.25 and $2.20 \mu \mathrm{m}$. Comparison to stellar population synthesis models indicates that if RD1 is a young $\left(t<10^{8} \mathrm{yr}\right)$ galaxy, significant reddening $\left(A_{V}>0.5 \mathrm{mag}\right)$ is indicated. Combined with observations of other high-redshift systems, these data show that dust is likely to be an important component of young galaxies even at redshifts of $z>5$. The extinction-corrected monochromatic luminosity of RD1 at $1500 \AA$ is then a factor of about 3 larger than $L_{1500}^{*}$ as determined by Dickinson for $z \sim$ 3 starburst galaxies. The implied star formation rate in RD1, corrected for extinction, is $\sim 50-100 h_{50}^{-2} M_{\odot} \mathrm{yr}^{-1}$.

Subject headings: dust, extinction — galaxies: evolution
\end{abstract}

\section{INTRODUCTION}

In the last several years, the combination of new wavelength dropout discovery techniques (e.g., Steidel et al. 1996) coupled with the deep imaging power of the Hubble Space Telescope and the spectroscopic capabilities of a new generation of large ground-based telescopes has lead to a blossoming of the study of galaxies at redshifts of $z>2$. A recent breakthrough in this field has been the discovery of a galaxy at a redshift of $z=$ 5.34 by Dey et al. (1998). Besides being the highest redshift object presently known, this galaxy is important because it is apparently neither gravitationally lensed nor the host of a powerful active nucleus. Thus, there is hope that detailed studies of this system and others like it at $z \sim 5$ will shed light on the intrinsic, stellar properties of very young galaxies.

Because visible observations of objects at such high redshifts are sampling the rest-frame ultraviolet part of the spectrum, they are very sensitive to the presence of even small amounts of intervening dust. Dust is important at these redshifts for three simple reasons. First, intrinsic luminosities and star formation rates are always underestimated when dust is not taken into account. This may have vast implications for metal production at early epochs (Madau et al. 1996; Meurer et al. 1997). Second, dust may hide entire populations of high-redshift, starforming galaxies from detection because of sample selection effects (e.g. Dey, Spinrad, \& Dickinson 1995). Third, dusty interstellar media (ISM) in galaxies at $z>3-4$, by their very nature, imply previous episodes of star formation and enrichment, pushing the first epoch of star formation to even higher redshifts. Although "pollution" of the ISM can happen relatively quickly from a truly primordial state (e.g., Matteucci \& Padovani 1993), dusty systems at high redshift always provide a lower limit to the redshift of the earliest star formation. Since there is mounting evidence for dust in high-redshift galaxies (e.g., Dunlop et al. 1994; van Ojik et al. 1994; Ivison 1995; Dey et al. 1995; Knopp \& Chambers 1997; Sawicki \& Yee

\footnotetext{
${ }^{1}$ Based on observations obtained at the W. M. Keck Observatory, which is operated jointly by the California Institute of Technology and the University of California.

${ }^{2}$ SIRTF Science Center, 100-22, California Intstitue of Technology, Pasadena, CA 91125.

${ }^{3}$ Palomar Observatory, 320-47, California Intstitue of Technology, Pasadena, CA 91125.
}

1998), it is natural to ask whether dust is present in galaxies at $z>5$.

To understand the true nature of the $z=5.34$ galaxy discovered by Dey et al. (1998), hereafter referred to as RD1 (for Red Dropout 1), it is necessary to know whether it is being viewed through a significant amount of dust. To achieve this, it is essential that such objects be observed at the longest possible wavelengths. We report in this Letter serendipitous observations made of this galaxy at $J(1.25 \mu \mathrm{m})$ and $K(2.20 \mu \mathrm{m})$. The galaxy is clearly detected at $J$, and simple fits to the observed optical-infrared colors suggest a reddened, young stellar population. In the discussion, we adopt the cosmological parameters $H_{0}=50 \mathrm{~km} \mathrm{~s}^{-1} \mathrm{Mpc}^{-1}$ and $q_{0}=0.5$. Throughout this Letter we refer to broadband magnitudes measured with respect to Vega, such that $J=0 \mathrm{mag}$ and $K=0 \mathrm{mag}$ correspond to 1578 and $646 \mathrm{Jy}$, respectively.

\section{OBSERVATIONS AND DATA REDUCTION}

As part of an observational study of high-redshift radio galaxies (Armus et al. 1998), we observed the most distant known radio galaxy, 6C 0140+32, in 1997 August and 1998 January using the near-infrared camera (NIRC) on the K1 telescope of the W. M. Keck Observatory. The instrument is described in detail by Matthews \& Soifer (1994). It has a $256 \times 256 \mathrm{InSb}$ array with $00^{\prime \prime} 15 \times 00^{\prime \prime} 15$ pixels for a $38^{\prime \prime} \times 38^{\prime \prime}$ field of view. On 1997 August 18, the field was observed at $K$ under conditions of thin cirrus and good seeing ( 0"5). On 1998 January 16 , we observed this field under clear, photometric conditions at $J$ and $K$ in excellent seeing (0"4-0".5 FWHM). On 1998 January 17 , we also observed the field at $K$ under conditions of good seeing with thin cirrus.

The target for the observations was centered in the field of view, and observations were made with individual integration times of $60 \mathrm{~s}$. In the August observations, the target was moved in a regular nine-point square grid covering a size $10^{\prime \prime}$ on a side, with the center of the grid moved by 1 " -2 " between grids. In the January observations, the target was moved randomly over an area of $10^{\prime \prime} \times 10^{\prime \prime}$ between individual observations in order to affect optimal sky subtraction.

Because the galaxy RD1 and the companion galaxy BD3 (for Blue Dropout 3) at a redshift $z=4.022$ were only $\sim 12$ " from the targeted object, they were contained in virtually every frame, and the combined data achieved the full sensitivity of 

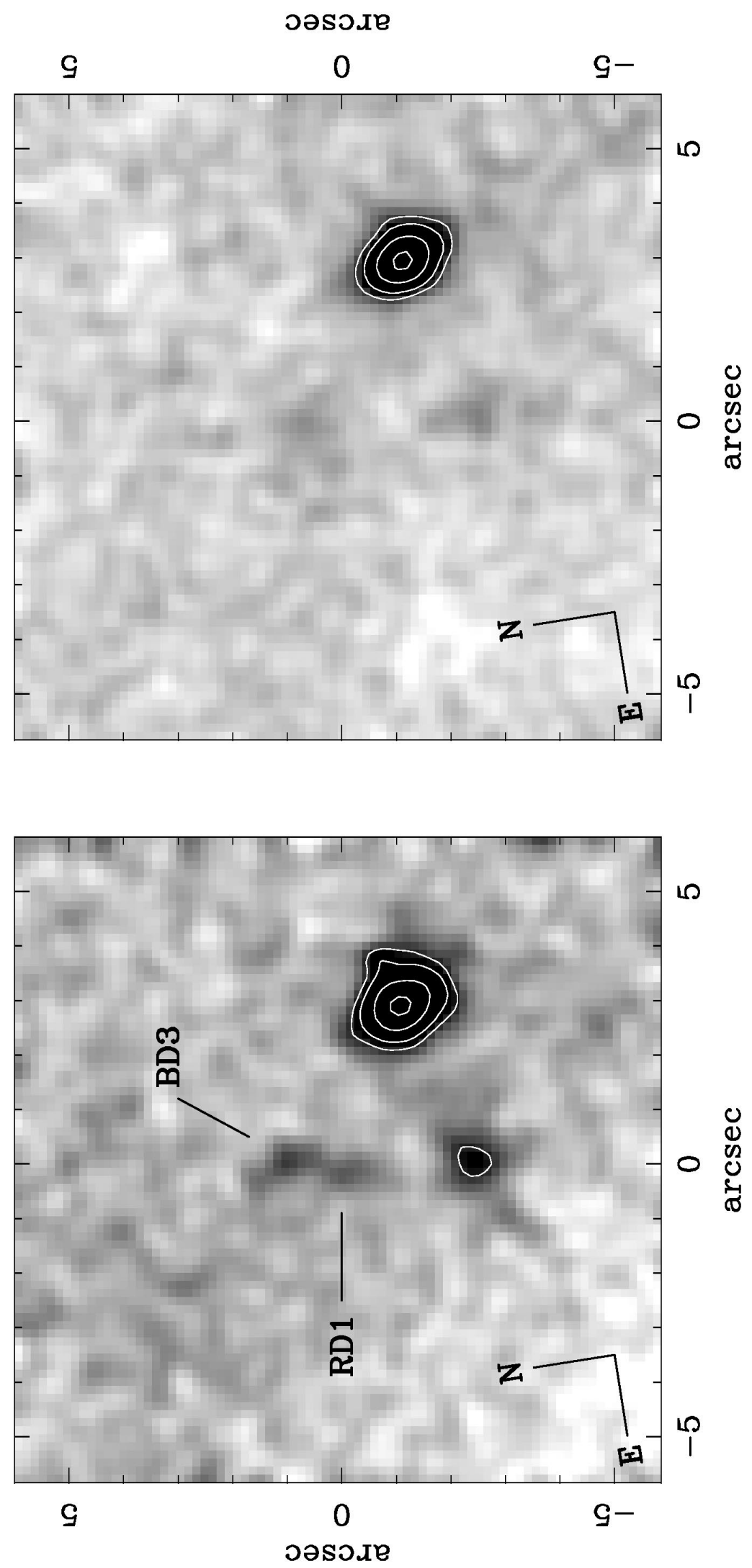
TABLE 1

Photometry OF RD1 AND BD3

\begin{tabular}{|c|c|c|c|}
\hline Object & $\begin{array}{c}I \\
(\mathrm{mag})\end{array}$ & $\begin{array}{c}J \\
(\mathrm{mag})\end{array}$ & $\begin{array}{c}K \\
(\mathrm{mag})\end{array}$ \\
\hline RD1 & $26.5 \pm 0.1^{\mathrm{ab}}$ & $24.3 \pm 0.3$ & $23.1^{\mathrm{c}}$ \\
\hline BD3 $\ldots . .$. & $25.1 \pm 0.1^{\mathrm{a}}$ & $24.1 \pm 0.3$ & $23.0 \pm 0.4$ \\
\hline \multicolumn{4}{|c|}{$\begin{array}{l}{ }^{a} I \text {-band magnitudes are from Dey et al. (1998) as } \\
\text { neasured in a } 1 \text { ". } 5 \text { diameter circular beam. } \\
\text { b The } I \text {-band magnitude of RD } 1 \text { has been corrected } \\
\text { or Ly } \alpha \text { emission in the bandpass as per Dey et al. } \\
{ }^{\mathrm{c}} 3 \sigma \text { limit, corresponding to a } 1 \sigma \text { limit of } 24.3 \\
\text { nag. }\end{array}$} \\
\hline
\end{tabular}

the observation at the location of RD1 and BD3 at each wavelength.

A bright star in the center of the NIRC field was used to accurately register the successive frames for co-addition. To remove time-variable fluctuations in illumination, separate sky and normalized flat-field frames were created from the data for each set of three images, by taking the median of the nearest seven to nine frames. After being trimmed to a size of $251 \times 251$ pixels, the individual data frames were skysubtracted, flat-fielded, and shifted to a common dc level after known bad pixels were flagged. These processed images were then aligned, using integer pixel shifts, and combined using a clipped mean algorithm. The FWHM of a point source in the final mosaic, as determined from several stellar images in the field, was approximately 0.5 at $J$ and 0.45 at $K$. The photometry was based soley on the data from 1998 January 16 when conditions were photometric. The data were calibrated by reference to the infrared standard stars of Persson et al. (1998).

\section{RESULTS}

The $J$ and $K$ images of the field centered on RD1 are shown in Figure 1. The $J$ data are from 1998 January 16 only, while the $K$ data are from 1998 August 18 and 1998 January 16 and 17. We obtained a total of 70 and 92 minutes of integration at $J$ and $K$, respectively.

In order to bring out the faint, high-redshift sources RD1 and BD3, the data in Figure 1 have been convolved with a circular Gaussian having an FWHM equal to the measured stellar FWHM in the final $J$ - and $K$-band mosaics. In this figure, $12^{\prime \prime}$ sections of the mosaics are displayed. Objects RD1 and BD3 are both detected in the $J$-band image. At $K$, BD3 is marginally detected, while RD1 is undetected. The $J$-band image of RD1 is resolved in the north-south direction, while that of BD3 is unresolved.

Because the objects RD1 and BD 3 are separated by $\sim 1$ ".2, obtaining photometry of both systems required special care. A square beam, 0.75 on a side, was centered on both RD1 and BD3. The sky was taken to be adjacent and to the south for RD1 and adjacent and to the north for BD3. As a check on this small-beam photometry, aperture photometry was obtained of the combined flux from both objects, and this total was distributed between the two objects based on the onedimensional distribution of flux along the line between the two sources. The two methods agreed well, and the magnitudes are reported in Table 1 . For RD1 we measure $J=24.3 \pm 0.3 \mathrm{mag}$ and $K>23.1 \mathrm{mag}$ ( $3 \sigma$ limit). For BD3 we measure $J=$ $24.1 \pm 0.3 \mathrm{mag}$ and $K=23.0 \pm 0.4 \mathrm{mag}$. In Table 1 we have also included the $I$-band magnitudes of these objects as reported by Dey et al. (1998).

To use the broadband magnitudes to derive continuum fluxes requires correction for contamination by strong emission lines contained within the filter bandpasses. In the case of RD1, the $J$ filter contains redshifted C III] $\lambda 1909$ (at $1.206 \mu \mathrm{m}$ ), while the $K$ filter contains redshifted [O II] $\lambda 3727$ (at $2.355 \mu \mathrm{m}$ ). Heckman et al. (1998) have recently compiled a composite UV spectrum of nearby starburst galaxies that shows the $\mathrm{C}$ III] emission-line equivalent width to be generally less than $10 \AA$. This is small compared with the uncertainty in the measured $J$-band magnitude of RD1. Similarly, the average radio galaxy spectrum in McCarthy (1993) can be used as an upper limit on the strength of $\mathrm{C}$ III] in RD1. If the equivalent width of C III] in RD1 is the same as in a typical radio galaxy (32 $\AA$ ), the C III] line would contribute only about $8 \%$ of the total flux in the $J$ filter. Thus, we conclude that the correction for line emission in the $J$ band for RD1 is not significant. However, unlike $\mathrm{C}$ III], the $\left[\mathrm{O}_{\mathrm{II}}\right] \lambda 3727$ line can be quite strong in starforming galaxies, having rest-frame equivalent widths of 50-100 A (e.g., Cowie, Hu, \& Songalia 1995; Gallagher, Bushouse, \& Hunter 1989). At an equivalent width of $100 \AA$, [O II] would contribute $14 \%$ of the $(3 \sigma)$ limit we measure for the $K$-band flux from RD1.

In the case of BD3, there are no strong lines expected to be present in the $J$ filter, unless $\mathrm{Mg}$ II at $2800 \AA$ has a broad, blue wing, which is unlikely for a starburst galaxy. The only emission feature that could contribute to the $K$-band measurement is the $\mathrm{H} \beta$ line. While potentially strong, the redshifted wavelength of $\mathrm{H} \beta(2.44 \mu \mathrm{m})$ places it at the very edge of the $K$ filter, and it should have a negligible effect on the total flux.

\section{DISCUSSION}

The most important result of these observations is that RD 1 was detected at $J$. This immediately implies that it is a much redder system than expected for any dust-free, young galaxy model. The expected color of an unreddened, star-forming galaxy with $f_{\nu} \sim$ const is $I-J \sim 0.5 \mathrm{mag}$ and $I-K \sim 1.5 \mathrm{mag}$, while the apparent color of RD1 is $I-J=2.2 \pm 0.3$ mag and $I-K<3.5 \mathrm{mag}$. Note that source BD3 is also redder than expected for an unreddened star-forming galaxy, having $I-$ $J=1.2 \pm 0.3 \mathrm{mag}$ and $I-K=2.2 \pm 0.5 \mathrm{mag}$.

For comparison, the colors of the galaxy CL $1358+62 \mathrm{G} 1$ at a redshift of $z=4.92$ (Franx et al. 1997; Soifer et al. 1998) are $I-J=1.2 \pm 0.1 \mathrm{mag}$ and $I-K=2.1 \pm 0.2 \mathrm{mag}$. For CL $1358+62 \mathrm{G} 1$, Soifer et al. have shown that galaxy models with reddenings of $0.1<E(B-V)<0.4$ mag provide substantially better fits to the overall energy distribution than do models with no reddening.

Similarly, Sawicki \& Yee (1998) find that the rest-frame UV-optical colors of spectroscopically confirmed $z>2$ Lyman break galaxies in the Hubble Deep Field (HDF) imply significant internal dust obscuration at these redshifts. Again, young populations with $E(B-V) \sim 0.3$ provide better fits to the data than do older, unreddened models. Although RD1 and BD3 appear redder than the six $z>3$ sources in Sawicki \& Yee, there is considerable scatter among the HDF galaxies. The range in $I-J$ color of the HDF galaxies (closest to the $J-$ $K$ colors of RD1 and BD3 in rest wavelength given the smaller redshifts of the Sawicki \& Yee galaxies) is $-0.98<I-J<$ $+0.65 \mathrm{mag}$, while the $J-K$ colors of RD1 and BD3 are less than +1.2 and $+1.1 \mathrm{mag}$, respectively. Since we have argued above that the $J$-band magnitude of RD1 is not likely to be significantly contaminated by strong line emission, it seems that the measured continuum colors of RD1 and BD3 are likely to be affected by dust. 
With only an $I-J$ color and a limit on the $I-K$ color, it is difficult to disentangle the effects of intrinsic galaxy color and reddening for RD1. However, we can identify a range of allowable ages and reddening values for a set of simple galaxy models by using the synthetic spectral energy distributions of Bruzual \& Charlot $(1993,1996)$. Because we are simply trying to set the constraints on reddening and stellar age, we choose to fit aging, instantaneous starburst models, since these are the intrinsically reddest galaxies at a given age, and thus they require the least amount of dust reddening to match a given observed spectrum. Continuous star formation models will always require more reddening by dust.

Before comparing the data to the models, a correction must be made to the observed $I$-band flux density for the presence of foreground absorption. Dey et al. (1998) correct their measured $I$-band magnitude for $\operatorname{Ly} \alpha$ emission and estimate $I=$ $26.5 \pm 0.1 \mathrm{mag}$ in a 1".5 diameter beam (see Table 1). However, there is no light detected blueward of Ly $\alpha$ in the spectrum, and much of this absorption may occur at redshifts well below $z=5.3$. Thus, the intrinsic rest-frame UV flux density of RD1 is underestimated if the $\operatorname{Ly} \alpha$ emission line alone is taken into account. By comparing the Keck I-band filter and CCD responses to the RD1 spectrum, we have estimated that this correction amounts to approximately $34 \%$ of the measured $I$-band light, assuming all the light blueward of $\operatorname{Ly} \alpha$ is absorbed in the intervening material. Once this is taken into account, RD1 has an $I$-band magnitude of $\sim 26.2 \mathrm{mag}$. It is this value that we use in all subsequent modeling.

The comparison of the present data with the Bruzual \& Charlot models shows that for RD1, either young, dusty models or "old," dust-free models provide acceptable fits to the $I-, J$-, and $K$-band data. We have used both solar and $0.2 Z_{\odot}$ metallicity models, although we consider the latter more representative of the galaxies at these redshifts. Both sets of models require significant absorption, $A_{V}>0.5 \mathrm{mag}$, for stellar populations with ages significantly less than $10^{8} \mathrm{yr}$. At an age of $10^{8} \mathrm{yr}$, the $0.2 Z_{\odot}$ model requires $A_{V}=0.3 \mathrm{mag}$, while the solar metallicity model requires no reddening to fit the data. Both sets of models deliver unacceptable fits to the data for ages of
$5 \times 10^{8}$ yr or greater, regardless of the reddening. In all cases, the Small Magellanic Cloud (SMC) reddening curve of Gordon \& Clayton (1998) and the Bruzual \& Charlot models with a Salpeter initial mass function have been used.

If RD1 is indeed a reddened, young galaxy, then both its luminosity and star formation rate are larger than those estimated by Dey et al. (1998). If RD1 has an age of significantly less than $10^{8} \mathrm{yr}$ and a metal abundance $\sim 0.2 Z_{\odot}$, the visual extinction is larger than $\sim 0.5 \mathrm{mag}$, and the extinction at 1270 $\AA$ is a minimum of $\sim 2.7$ mag, based on the reddening curve for the SMC (Gordon \& Clayton 1998). Assuming the reddening to the Ly $\alpha$ line is the same as that to the far-UV continuum, the Ly $\alpha$ and UV continuum luminosity are larger by factors of 10-15 than those determined by Dey et al. (1998) for no reddening. The monochromatic luminosity of RD1 at $1500 \AA$ is then a factor of $\sim 3$ larger than $L_{1500}^{*}$ determined by Dickinson (1998) for $z \sim 3$ starburst galaxies. The star formation rate in RD1 is then $\sim 50-100 h_{50}^{-2} M_{\odot} \mathrm{yr}^{-1}$, which is comparable to the extinction-corrected values estimated by Sawicki \& Yee for the $z>2$ Lyman break galaxies in the HDF.

We thank R. Goodrich for assistance with the observations and S. E. Persson for providing photometric standards in advance of publication. Discussions with Daniela Calzetti, Arjun Dey, Tim Heckman, and David Hogg are greatly appreciated. Mike Pahre provided us with the Keck I-band filter and CCD response curves, and we thank him for that. We also would like to thank the referee, Hy Spinrad, for a critical reading of the manuscript and a number of valuable suggestions. The W. M. Keck Observatory is operated as a scientific partnership between the California Institute of Technology, the University of California, and the National Aeronautics and Space Administration. It was made possible by the generous financial support of the W. M. Keck Foundation. Infrared astronomy at Caltech is supported by grants from the NSF and NASA. This research has made use of the NASA/IPAC Extragalactic Database, which is operated by the Jet Propulsion Laboratory, Caltech, under contract with NASA.

\section{REFERENCES}

Armus, L., Soifer, B. T., Murphy, T. W., Jr., Neugebauer, G., Evans, A. S., \& Matthews, K. 1998, ApJ, 495, 276

Bruzual, G., \& Charlot, S. 1993, ApJ, 405, 538 1996, GISSEL Population Synthesis Models

Cowie, L. L., Hu, E., \& Songalia, A. 1995, Nature, 377, 603

Dey, A., Spinrad, H., \& Dickinson, M. 1995, ApJ, 440, 515

Dey, A. J., Spinrad, H., Stern, D., Graham, J. R., \& Chaffee, F. H. 1998, ApJ, 498, 93

Dickinson, M. 1998, in The Hubble Deep Field, Proc. STScI 1997 May Symp., ed. M. Livio, S. M. Fall, \& P. Madau (Cambridge: Cambridge Univ. Press), in press

Dunlop, J. S., Hughes, D. H., Rawlings, S., Eales, S., \& Ward, M. J. 1994, Nature, 370, 347

Franx, M., Illingworth, G. D., Kelson, D. D., van Dokkum, P. G., \& Tran, K.-V. 1997, ApJ, 486, L75

Gallagher, J. S., Bushouse, H., \& Hunter, D. A. 1989, AJ, 97, 700

Gordon, K. D., \& Clayton, G. C. 1998, ApJ, 500, 816

Heckman, T. M., Robert, C., Leitherer, C., Garnett, D. R., \& van der Rydt, F. 1998, preprint
Ivison, R. J. 1995, MNRAS, 275, L33

Knopp, G. P., \& Chambers, K. C. 1997, ApJ, 487, 644

Madau, P., Ferguson, H. C., Dickinson, M. E., Giavalisco, M., Steidel, C. C., \& Fruchter, A. 1996, MNRAS, 283, 1388

Matteucci, F., \& Padovani, P. 1993, ApJ, 419, 485

Matthews, K., \& Soifer, B. T. 1994, Infrared Astronomy with Arrays: The Next Generation, ed. I. McLean (Dordrecht: Kluwer), 239

McCarthy, P. J. 1993, ARA\&A, 31, 639

Meurer, G. R., Heckman, T. M., Lehnert, M. D., Leitherer, C., \& Lowenthal, J. 1997, AJ, 114, 54

Persson, S. E., Murphy, D. C., Krzeminski, W., Roth, M., \& Rieke, M. 1998, AJ, submitted

Sawicki, M., \& Yee, H. K. C. 1998, ApJ, 115, 1329

Soifer, B. T., Neugebauer, G., Franx, M., Matthews, K., \& Illingworth, G. D. 1998, ApJ, 501, L171

Steidel, C. C., Giavalisco, M., Pettini, M., Dickinson, M., \& Adelberger, K. L. 1996, ApJ, 462, L17

van Ojik, R., Rottgering, H. J. A., Miley, G. K., Bremer, M. N., Macchetto, F., \& Chambers, K. C. 1994, A\&A, 289, 54 